\title{
Il gradiente implicito nella verifica a fatica di giunzioni saldate sollecitate a fatica
}

\author{
R. Tovo, P. Livieri \\ Università di Ferrara, Dipartimento di Ingegneria, via Saragat 1, 44100 Ferrara, roberto.tovo@unife.it
}

RIASSUNTO. L'incremento delle potenzialità di strumenti per la progettazione assistita (come modellatori solidi e strumenti per FEA in grado di gestire modelli molto complessi) permette di ipotizzare lo sviluppo di strumenti numerici specifici per la previsione della resistenza a fatica delle giunzioni saldate. Tali strumenti potrebbero essere in grado di valutare l'influenza di geometria e carichi senza la necessità di elaborazioni successive, e spesso, del progettista (come nelle tensioni di Hot Spot).

Il presente lavoro propone una metodologia di calcolo adatta alla previsione della vita a fatica di giunzioni saldate complesse. Un indice di resistenza è ottenuto innanzitutto risolvendo il problema tensionale completamente in modo numerico (agli elementi finiti). La previsione della resistenza a fatica, è calcolata facendo uso di un modello analitico basato sul gradiente implicito che assume come tensione efficace la tensione equivalente non locale derivante dalla tensione principale.

Dapprima verrà tarato il metodo su prove sperimentali eseguite su giunzioni saldate a croce, successivamente il metodo verrà utilizzato per la verifica a fatica di giunzioni saldate più complesse a sviluppo tridimensionale.

\begin{abstract}
In this paper, a non-local equivalent stress is calculated by solving a second order differential equation of implicit type. The solution is obtained by assuming a linear elastic constitutive behaviour and the maximum principal stress as equivalent stress. Fatigue behaviour of steel welded joints is taken into account and a general fatigue scatter band is proposed. The non local stress is computed, namely the effective stress, by means of a completely numerical solution of local elastic stress field. In complex 3D welded details the critical point turns out from the analysis and it is not assumed a priori.
\end{abstract}

Parole chiave. Fatica, giunti saldati, tensioni non-locali.

\section{INTRODUZIONE}

I 1 disegno e la modellazione tridimensionale assistiti negli ultimi anni sono diventati, in molte aziende del settore meccanico, uno standard di progettazione che offre il vantaggio di cogliere l'aspetto e il funzionamento nelle tre dimensioni nonché la possibilità di usare la formulazione matematica dei volumi considerati per ulteriori elaborazioni. $\mathrm{Nel}$ caso delle saldature, ed in particolare dei cordoni d'angolo, nella rappresentazione tridimensionale diviene naturale la schematizzazione con figure prismatiche o di rivoluzione aventi spigoli vivi. Tale assunzione è di fatto vicina alla realtà in quanto i cordoni di saldatura ottenuti ad arco, sono caratterizzati da raggi di raccordo il cui valore medio è nell'ordine di grandezza di qualche decimo di millimetro [1-3]. Se da una parte, l'adozione di uno spigolo vivo può semplificare la modellazione del componente, dall'altra, dal punto di vista dell'analisi strutturale, si introduce nel campo tensionale una singolarità (ossia un punto con soluzione tendente all'infinito) che impedisce l'uso di modelli di verifica basati sulla imposizione di un limite tensionale. L'idea di confrontare un limite ritenuto ammissibile per il materiale con il valore di picco del campo di tensione, non può essere adottato in quanto qualunque valore assunto per la tensione ammissibile viene inevitabilmente superato purché ci si avvicini sufficientemente allo spigolo.

In queste situazioni la letteratura scientifica a volte consiglia di utilizzare le tensioni nominali o le tensioni di hot spot [4]. Purtroppo le prime (nominali) spesso non sono definite o calcolabili in modelli solidi geometricamente complessi. Le tensioni di hot spot hanno altri problemi: il primo è che necessitano di un post-processing manuale e spesso non chiaro 
nella procedura da seguire; inoltre le previsioni sono a volte imprecise (approssimative), non valutano l'effetto scala o non sono in grado di predire il comportamento meccanico di punti di rottura diversi dal piede del cordone (come i cedimenti alla radice del cordone).

Per una trattazione teorica degli aspetti matematici e geometrici, in presenza di un raggio di raccordo nullo dei cordoni di saldatura, è possibile affidarsi ad approcci locali basati sul calcolo dei Notch Stress Intensity Factors (NSIF) valutati in prossimità dei punti in cui innesca la cricca per fatica [6-11]. Nel caso in cui solo modo I sia singolare o di modo I predominante su modo II, l'NSIF può essere usato direttamente per il calcolo della vita a fatica utilizzando specifiche bande di dispersione $[7,8,11]$, mentre in condizioni di modo misto di sollecitazione, o nell'ottica di utilizzare un'unica banda di dispersione valida per qualunque angolo di apertura del cordone di saldatura, si rende necessario l'impiego di un parametro di validità più generale come, ad esempio, l'energia media all'interno di un settore circolare posto in prossimità del punto critico [9-11].

Per gli aspetti più applicativi sarebbe opportuno disporre di un approccio metodologico che sia congruente con l'inquadramento agli NSIF per l'analisi del comportamento a fatica, ma al contempo adatto all'impiego per un computo interamente numerico del problema della resistenza a fatica indipendentemente dalla complessità del giunto senza assumere a priori il punto di innesco della cricca. Ossia l'obiettivo è quello di proporre una metodologia, adatta al calcolo della resistenza a fatica delle giunzioni saldate e capace, indipendentemente della complessità del giunto, di riportare la verifica a fatica al calcolo di un valore "efficace" della tensione calcolabile con strumenti automatici integrati con solutori agli elementi finiti.

La presente memoria presenta una possibile soluzione del problema metodologico attraverso l'approccio denominato "gradiente implicito" [12-14].

Per validare il metodo proposto, saranno considerati dati sperimentali presi dalla letteratura di giunzioni saldate analizzabili con schemi bidimensionali, altresì a dettagli strutturali complessi schematizzati con modelli tridimensionali.

\section{MODELLO NON LOCALE}

A ssegnato un corpo generico di volume $\mathrm{V}$, in accordo con le referenze [15] e [16] è possibile definire una tensione equivalente non locale $\bar{\sigma}$ nel punto generico $P$ del volume $\mathrm{V}$ come media integrale di una tensione equivalente locale $\sigma_{\text {eq }}$ pesata con una opportuna funzione $\alpha$ che tiene in considerazione la distanza $s$ del generico punto $Q$ dal punto $P$ dei punti del volume $\mathrm{V}(\mathrm{s}=|\mathrm{PQ}|)$ :

$$
\bar{\sigma}(\mathrm{P})=\frac{1}{\mathrm{~V}_{\mathrm{r}}(\mathrm{P})} \int_{\mathrm{V}} \alpha(|\mathrm{PQ}|) \sigma_{\text {eq }}(\mathrm{Q}) \mathrm{dV} \quad \text { in } \mathrm{V}
$$

Nell'equazione (1), il simbolo $\mathrm{V}_{\mathrm{r}}(\mathrm{P})$ denota il volume di riferimento calcolato come $\mathrm{V}_{\mathrm{r}}(\mathrm{P})=\int_{\mathrm{V}} \alpha(|\mathrm{PQ}|) \mathrm{dV}$.

Senza entrare nel dettaglio dei modelli non locali, il problema del calcolo della tensione equivalente non locale $\bar{\sigma}$ può essere trasferito alla risoluzione di una equazione differenziale del secondo ordine [17]. Dopo aver assunto la $\bar{\sigma}$ come tensione efficace ai fini della resistenza a fatica $\left(\sigma_{\text {eff }}\right)$, l'integrale (1) equivale a risolvere la seguente equazione differenziale:

$$
\sigma_{\text {eff }}-c^{2} \nabla^{2} \sigma_{\text {eff }}=\sigma_{\text {eq }} \quad \text { in } \mathrm{V}
$$

dove $c$ è una dimensione intrinseca legata al materiale in esame, $\nabla^{2}$ è l'operatore di Laplace e $\sigma_{\text {eq }}$ è la tensione equivalente locale ritenuta responsabile del danno a fatica (per una trattazione più approfondita del problema si rimanda alle referenze [12-14]).

\section{Calcolo Della tensione efFicace}

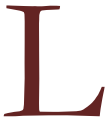

'obiettivo del presente lavoro è quello di risolvere l'equazione differenziale (2) in presenza di singolarità tensionali indotte dai cordoni di saldatura pensati come intagli ideali a spigolo vivo. A tale scopo, è stata messa a punto una procedura di tipo numerico capace di risolvere il problema (2) della non-località della tensione in modo completamente automatico. 
Nel caso di sollecitazione a fatica con carichi in fase, le direzioni principali rimangono invariate nel tempo ed il calcolo a fatica può risolversi facendo riferimento ai soli valori minimi e massimi della tensione efficace. In particolare, per le saldature che non hanno subito un trattamento di distensione termica, è sufficiente far riferimento al solo range della tensione efficace $\left(\Delta \sigma_{\text {eff }}\right.$. Perciò, per le saldature, nella (2) andranno inserite le variazioni $\Delta$ che subiscono le tensioni nel ciclo di fatica.

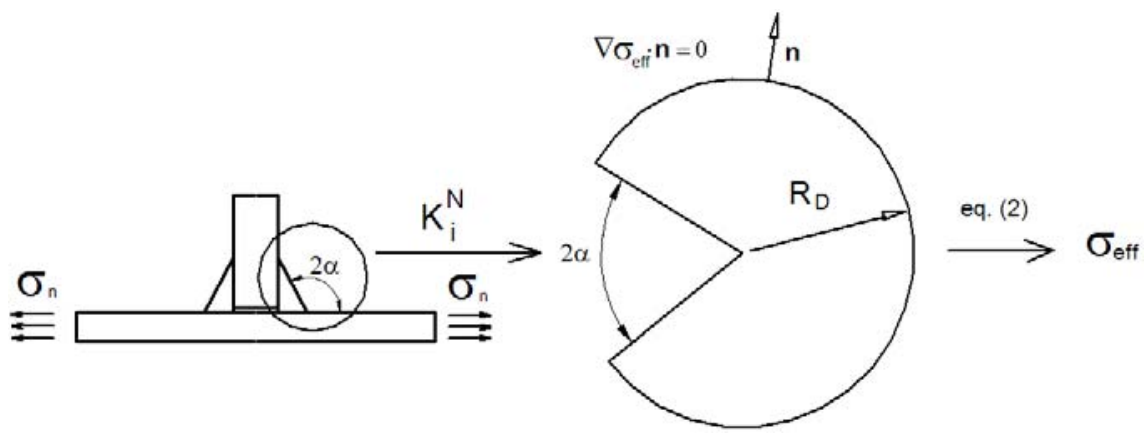

Figura 1: Geometria di riferimento.

\section{Solurione numerica 2D}

Alcune tipologie di giunti saldati come i giunti a croce e i giunti a $\mathrm{T}$, possono essere studiati come giunzioni a sviluppo bidimensionale ipotizzando nulli i gradienti di tensione nella terza dimensione.

Noto il parametro $c$ del materiale in esame e le condizione al bordo, è possibile risolvere l'equazione ellittica (2) nell'intero volume $\mathrm{V}$ in funzione della tensione equivalente $\sigma_{\text {eq }}$ scelta. Espressioni asintotiche della tensione $\sigma_{\text {eq }}$ equivalente possono essere valutate se si conoscono a priori i valori dei Notch Stress Intensity Factors (NSIF) nel punto in cui innesca la cricca per fatica [13]. Sfruttando un sistema di riferimento polare, la soluzione numerica può essere ottenuta su un settore circolare di raggio $\mathrm{R}_{\mathrm{D}}$ di dimensioni maggiori al parametro $c$ del materiale $\left(c<<\mathrm{R}_{\mathrm{D}}\right)$. La Fig. 1 mostra il dominio di integrazione e le condizioni al bordo applicate. Come condizioni al bordo, in [13] sono state prese in esame solo le condizioni di Neumann che esprimono l'ortogonalità del gradiente della soluzione cercata con la normale uscente al bordo del dominio di integrazione (dal punto di vista fisico ciò equivale ad imporre un flusso nullo della soluzione in direzione ortogonale al bordo). Ciò nonostante, se non sono noti gli NSIF della saldatura si può comunque ricorrere ad una soluzione completamente numerica del problema differenziale.

La Fig. 2, riporta un esempio di calcolo della tensione $\Delta \sigma_{\text {eff }}$ (adimensionalizzata rispetto alla tensione nominale nel piatto più spesso) ottenuta a partire da una analisi lineare elastica del problema tensionale che vede nei sei punti indicati in figura una singolarità del campo di tensione in accordo con la soluzione di Williams [18]. In Fig. 2, come tensione equivalente $\sigma_{\mathrm{eq}}$ locale è stata assunta la tensione principale massima. Appare evidente che la singolarità del campo di tensione di partenza viene smussata, ottenendo un campo di tensione equivalente non locale di tipo continuo su tutto il giunto. La sola analisi della tensione efficace riportata come grafico tridimensionale al di sopra del dominio di integrazione, fornisce contemporaneamente la posizione di probabile innesco del difetto per fatica ed il valore del picco della tensione efficace. Va comunque sottolineato che a sostegno di quanto calcolato numericamente, accurate soluzioni analitiche di casi monodimensionali e bi-dimensionali sono riportate nelle referenze $[12,13]$.

\section{Soluzione numerica 3D}

Nel caso di strutture saldate complesse per le quali non sia possibile ridursi ad uno schema di calcolo bidimensionale o nel caso in cui gli NSIF non siano noti, la procedura presentata al punto Soluzione numerica 2D non può essere applicata. Per superare il problema, è stata messa a punto una procedura di calcolo integrata, che partendo da un modello CAD 3D della saldatura è capace di risolvere il problema del calcolo della tensione efficace $\sigma_{\text {eff }}$ sull'intero componente. Il problema della realizzazione di una mesh sufficientemente accurata sia per il calcolo tensionale che per la risoluzione del sistema differenziale (2), può essere gestito in modo completamente automatico con mesh di tipo adattativo. In questo modo non è richiesta a priori la conoscenza del punto di innesco della cricca da parte dell'operatore ma è necessario fornire solamente la geometria, i vincoli ed i carichi esterni agenti. Le Fig. 3 e 4 mostrano esempi di modelli CAD e delle relative mesh 3D, ad elementi tetraedrici, adatte per la risoluzione dell'Eq. (2). Nelle figure vine anche illustrato il campo

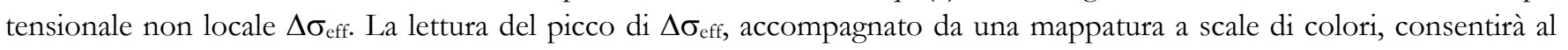


progettista di localizzare la zona critica della connessione saldata permettendo inoltre di calcolare il coefficiente di sicurezza o la vita a fatica del particolare saldato sulla base delle curva di progetto riportate nei successivi capitoli.

Gli esempi riportati nel seguito mostreranno l'efficienza del metodo e la sua capacità di prevedere la resistenza a fatica di strutture complesse tridimensionali.

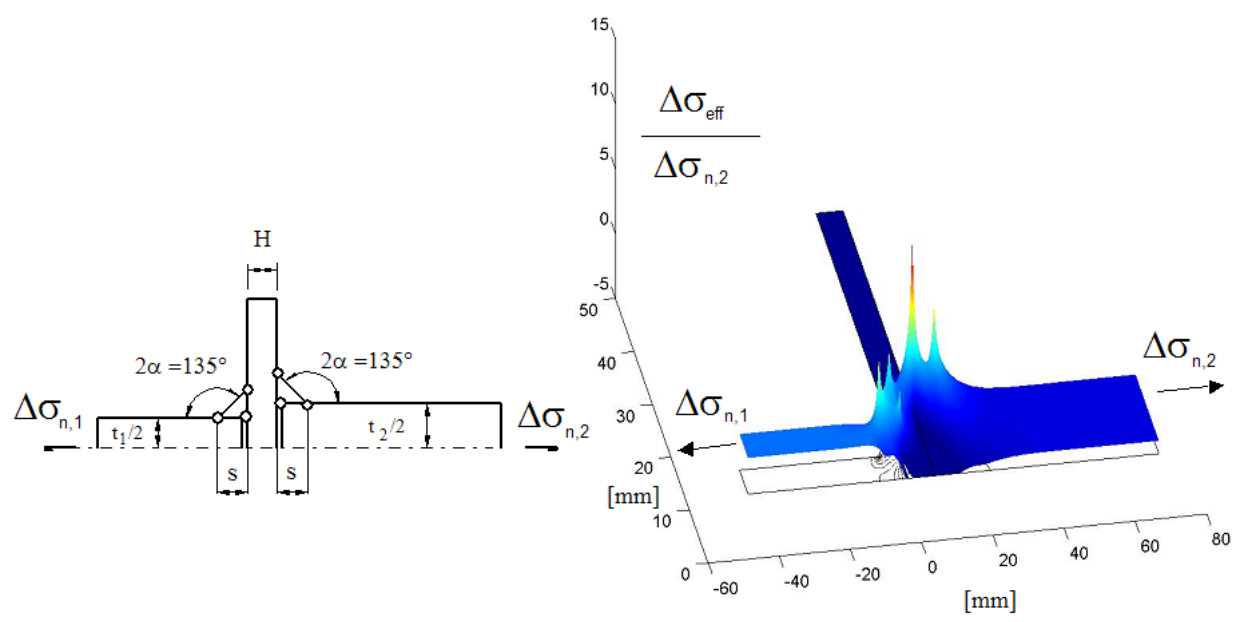

Figura 2: Tipica soluzione in termini di tensione efficace di una giunzione piana a cordone portante.

La tensione non locale è adimensionalizzata rispetto alla tensione nominale nel piatto di maggiore spessore.

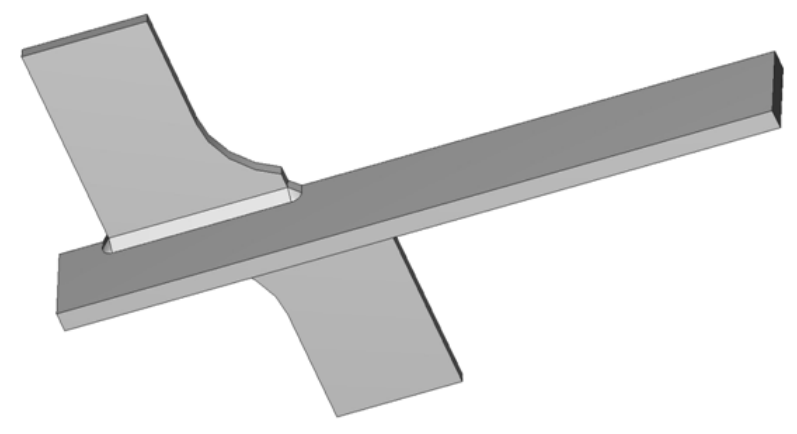

(a)

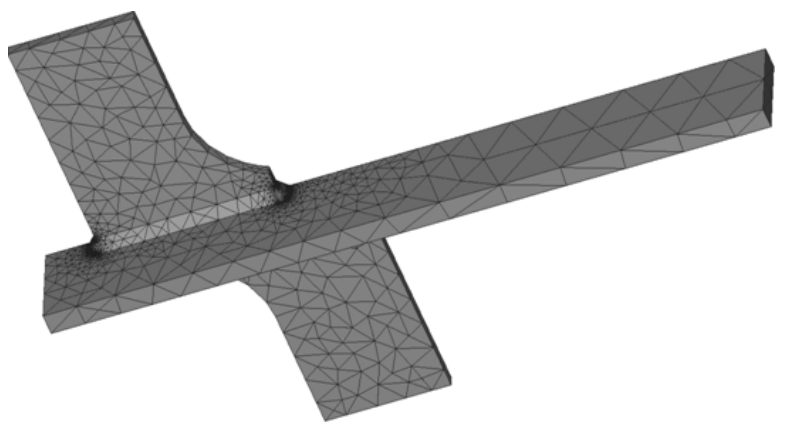

(b)

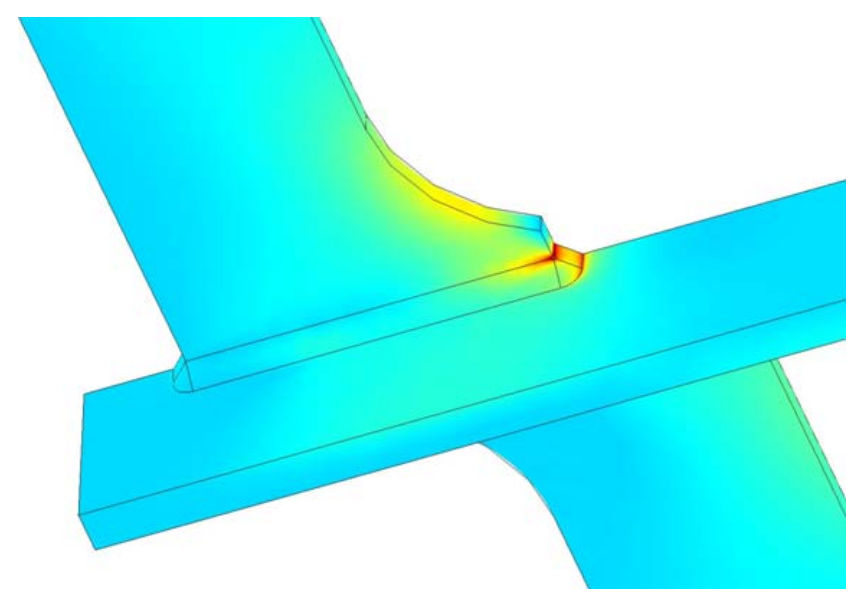

(c)

Figura 3: Esempio di mesh e soluzione ottenuto in automatico partendo dal modello CAD di un giunto complesso:

a) Modello CAD di un giunto a cordone portante sollecitato a trazione sul piatto principale.

b) Mesh ad elementi tetraedrici utilizzata per analizzare il giunto;

c) Mappatura a scale di colori della tensione efficace (massima tensione principale come tensione equivalente non locale; $\mathrm{c}=0.2 \mathrm{~mm}$ ). 


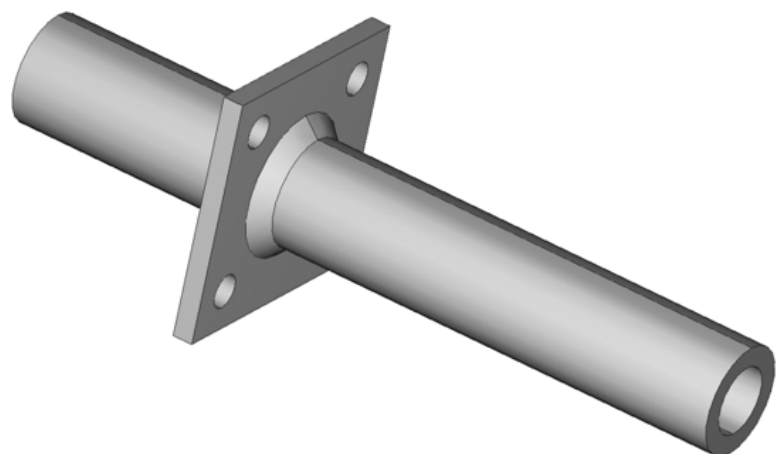

(a)

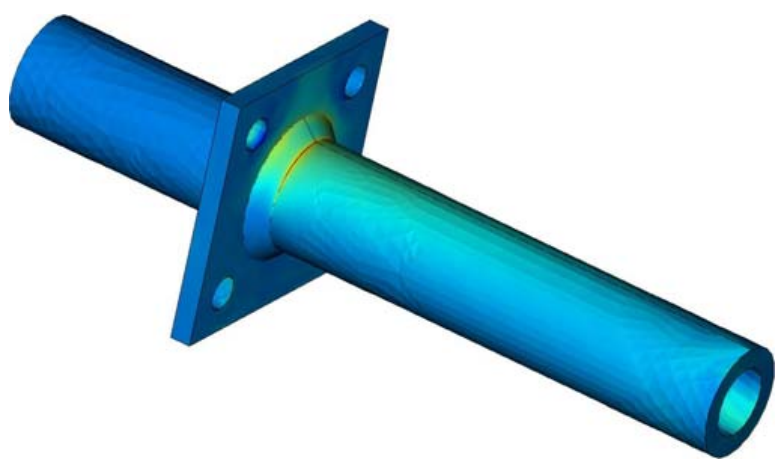

(c)

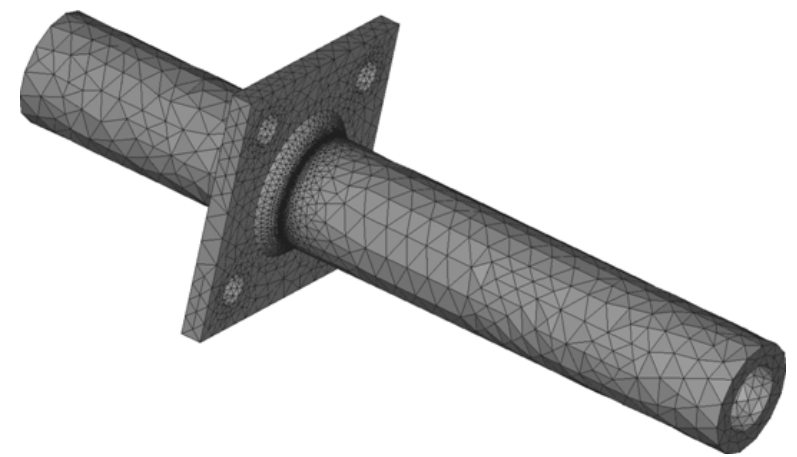

(b)

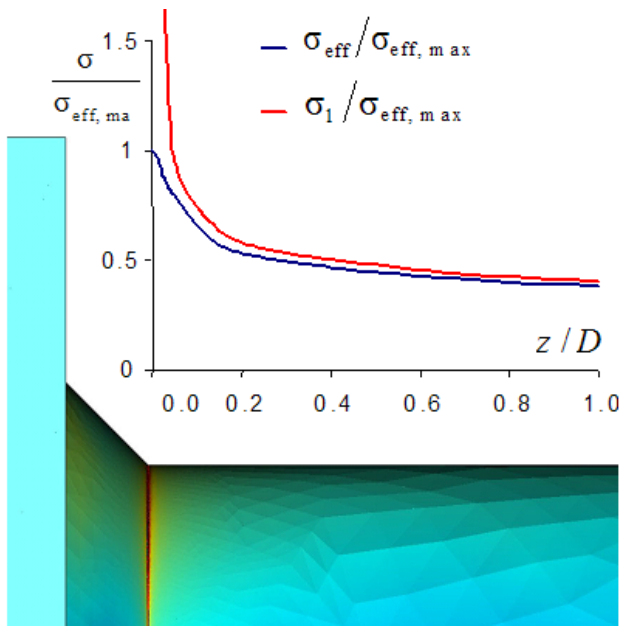

(d)

Figura 4: Esempio di mesh e soluzione ottenuta in automatico partendo dalla geometria del giunto. a) Modello CAD di un giunto a cordone portante sollecitato a flesso-torsione. b) Mesh ad elementi tetraedrici utilizzata per analizzare il giunto; c) Mappatura a scale di colori della tensione efficace (massima tensione principale come tensione equivalente non locale; $\mathrm{c}=0.2 \mathrm{~mm}$ ); $\mathrm{d}$ ) Andamento della tensione principale massima $\sigma_{1}$ e della tensione efficace $\sigma_{\text {eff }}$ lungo una generatrice esterna del cilindro. I valori sono normalizzati rispetto al valore massimo della tensione efficace $\sigma_{\text {eff, max }}$ e sono rappresentati in funzione della distanza $z$ dal piede del cordone normalizzata rispetto al diametro esterno del tubo D.

\section{VALUTAZIONE DEL PARAMETRO $C$ PER LE SALDATURE}

$\mathrm{I}$ potizzato che il parametro $c$ dipenda solamente dal materiale, è possibile calcolarne il suo valore numerico confrontando la resistenza a fatica, in termini di variazione della tensione efficace $\Delta \sigma_{\text {eff, di saldature con rotture alla }}$ radice $\left(2 \alpha=0^{\circ}\right)$ con saldature aventi rotture al piede $\left(2 \alpha=135^{\circ}\right)$. In accordo con la referenza [12], il valore di $c$ per le saldature ad arco in acciaio è di circa $0.2 \mathrm{~mm}\left(\mathrm{c}^{2}=0.04 \mathrm{~mm}^{2}\right)$. D'altra parte, analisi numeriche [12] e soluzioni analitiche [13] hanno evidenziato che $c$ è legato attraverso una costante di proporzionalità ₹ alla distanza critica di El-Haddad et al. [21], definita dal limite di fatica del provino liscio $\sigma_{\mathrm{o}}$ e dal valore di soglia dello Stress Intensity Factor $\Delta \mathrm{K}_{\mathrm{th}}$ :

$$
\mathrm{a}_{0}=\frac{1}{\pi}\left(\frac{\Delta \mathrm{K}_{\mathrm{th}}}{\Delta \sigma_{0}}\right)^{2}
$$

Perciò, in un caso generale noto $\mathrm{a}_{0}$ risulta immediato il calcolo di $c$ in relazione alla tensione equivalente assunta. La relazione fra a $\mathrm{a}_{0} \mathrm{e} c$ può essere così riassunta [12]:

$$
\mathrm{c}=\mathrm{za}_{0}
$$

La Tab. 1 propone il valore di ₹ per le diverse tensioni equivalenti. 


\begin{tabular}{|c|c|c|}
\hline $\begin{array}{l}\text { Tensione equivalente } \\
\text { locale }\end{array}$ & $\begin{array}{c}\text { Tipo di } \\
\text { sollecitazione }\end{array}$ & $\begin{array}{c}\mathrm{z} \\
\mathrm{c}=\mathrm{za}_{\mathrm{o}}\end{array}$ \\
\hline massima principale & $\begin{array}{l}\text { Stato piano di } \\
\text { tensione }\end{array}$ & 0.545 \\
\hline von Mises & $\begin{array}{l}\text { Stato piano di } \\
\text { tensione }\end{array}$ & 0.456 \\
\hline Tresca & $\begin{array}{l}\text { Stato piano di } \\
\text { tensione }\end{array}$ & 0.545 \\
\hline massima principale & $\begin{array}{l}\text { Stato piano di } \\
\text { deformazione }\end{array}$ & 0.545 \\
\hline von Mises & $\begin{array}{l}\text { Stato piano di } \\
\text { deformazione }\end{array}$ & 0.224 \\
\hline Tresca & $\begin{array}{l}\text { Stato piano di } \\
\text { deformazione }\end{array}$ & 0.267 \\
\hline
\end{tabular}

Tabella 1: Valori del parametro z per differenti tensioni equivalenti [12].

\section{BANDA DI DISPERSIONE PER LE SALDATURE E CURVA DI PROGETTO}

I 1 metodo del gradiente implicito consente di rendere continuo un campo di tensione singolare anche nell'ipotesi di materiale lineare elastico. Con tale formulazione è possibile, utilizzare il picco di tensione direttamente per il calcolo del coefficiente di sicurezza senza incorrere in errori formali. Utilizzando dati sperimentali di letteratura è possibile tracciare una banda di dispersione per le saldature nel campo della vita a termine fra $10^{4}$ e 5.106 cicli. Con riferimento alle serie sperimentali precedentemente analizzati nelle referenze[7, 8, 11], in Fig. 4 è stata tracciata la banda di dispersione in termini di variazione della tensione equivalente non locale massima $\Delta \sigma_{\text {eff,max }}$ calcolata in prossimità del punto di innesco della cricca. Gli spessori del piatto principale e degli irrigidimenti variavano da 3 a $100 \mathrm{~mm}$. Il valore della pendenza della curva di Wöhler risulta pari a 3 ed il valore di riferimento a $2 \cdot 10^{6}$ cicli al 97.7\% di probabilità di sopravvivenza è di 151 MPa.

In Fig. 4 è possibile osservare che la curva di progetto proposta dall'Eurocodice 3 [5] per i particolari tagliati all'ossitaglio automatico presenta una classe di resistenza $\left(140 \mathrm{MPa}\right.$ a $\left.2 \cdot 10^{\circ}\right)$ di poco inferiore al valore ottenuto per una probabilità di sopravvivenza del $97.7 \%(151 \mathrm{MPa})$.

Perciò, in analogia con l'Eurocodice 3 potremo dire che le giunzioni saldate, indipendentemente dalla loro forma, sollecitate principalmente a modo I, ricadono tutti all'interno di una stessa classe, quantificabile in circa $150 \mathrm{MPa}$ con pendenza fra $10^{4}$ e $5 \cdot 10^{6}$ cicli pari a 3 .

\begin{tabular}{ccccc}
\hline Caso & $\begin{array}{c}\mathrm{s} \\
{[\mathrm{mm}]}\end{array}$ & $\begin{array}{c}\mathrm{h} \\
{[\mathrm{mm}]}\end{array}$ & $\begin{array}{c}\mathrm{t}_{1} \\
{[\mathrm{~mm}]}\end{array}$ & $\begin{array}{c}\mathrm{t}_{2} \\
{[\mathrm{~mm}]}\end{array}$ \\
1 & 6 & 14 & 14 & 14 \\
2 & 6 & 14 & 9 & 14 \\
3 & 6 & 6 & 9 & 14 \\
4 & 6 & 6 & 9 & 25 \\
\hline
\end{tabular}

Tabella 2: Valori dei parametri geometrici di Fig. 2.

\section{ESEMPI APPLICATIVI}

$\mathrm{I}$ 1 problema differenziale (2), tranne nel caso di una cricca su una piastra di dimensione infinita [13], non è di semplice soluzione e comunque impone la conoscenza degli NSIF. Perciò ai fini progettuali risulta sicuramente molto efficiente una soluzione completamente numerica del problema differenziale. A tale scopo, è stata messa a punto una procedura di calcolo che richiede all'operatore come dati di ingresso la geometria del giunto e la costante $c$ del materiale 
ignorando completamente il problema della singolarità in prossimità degli intagli acuti ed affidando il problema della convergenza a soluzioni di tipo adattivo.

\section{Giunzioni a T sollecitate a flessione}

In Fig. 5, sono riportati i valori sperimentali di rotture avvenute al piede del cordone di saldatura per una giunzione avente un angolo diverso da quello esaminato in Fig. 4. I risultati numerici contrassegnati dal simbolo sono relativi ad un giunto a $\mathrm{T}$ sollecitato a flessione con angolo di apertura del cordone di $118^{\circ}$. Tali punti vanno a cadere all'interno della banda di dispersione precedentemente calcolata.
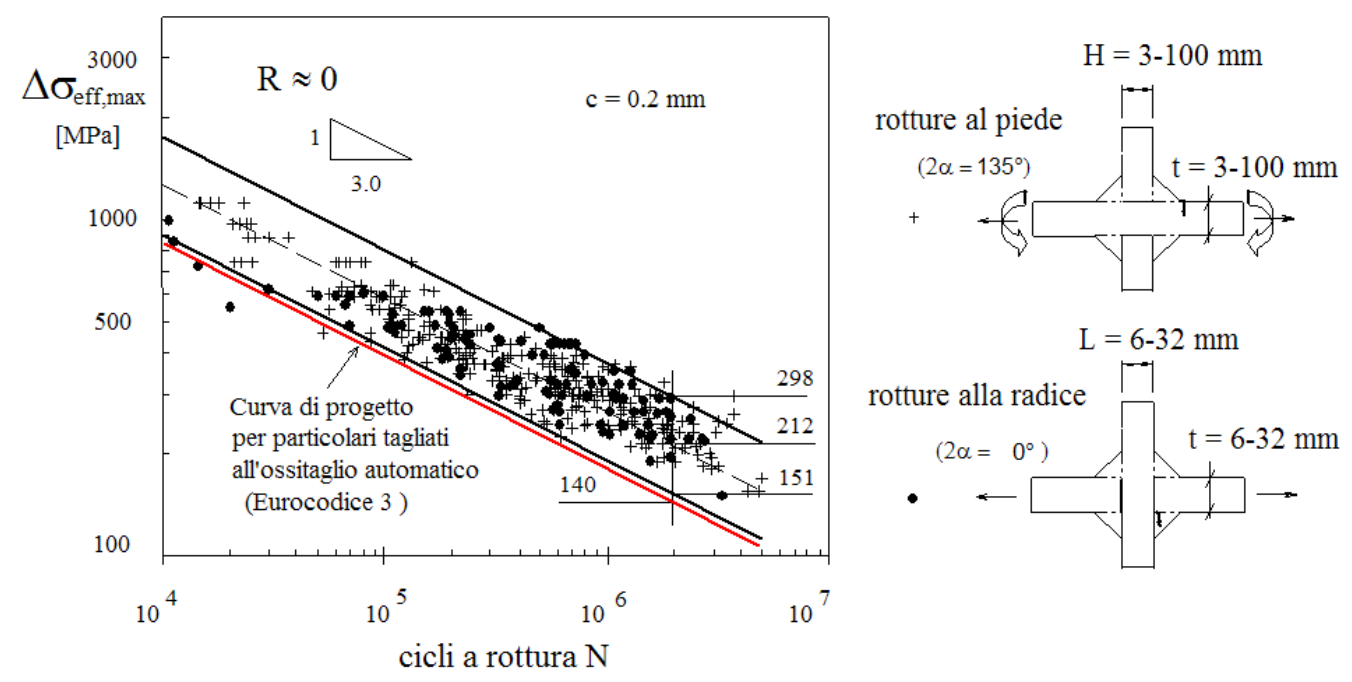

Figura 5: Banda di dispersione per giunzioni saldate ad arco in acciaio in termini di tensione efficace massima ottenuta con il metodo del gradiente implicito ( $R$ rapporto di ciclo)

\section{Previsione della zona di innesco della cricca per giunzioni a croce a cordone portante sollecitare a trazione}

Un altro interessante esempio è quello relativo allo studio del punto di innesco della cricca per fatica in un giunto a croce a cordone portante sollecitato a trazione in cui gli spessori dei piatti principali siano diversi [22]. La Fig. 6 mostra la geometria del giunto in esame mentre le dimensioni geometriche delle 4 serie analizzate nella referenza [22] sono riportate in Tab. 2. I punti critici di probabile innesco della cricca per fatica sono i punti evidenziati con un "cerchio" in Fig. 2.. Nonostante le differenze geometriche fra le quattro serie di Tab. 2, dal punto di vista sperimentale si sono sempre osservate rotture alla radice del cordone di saldatura dalla parte piatto più spesso.

I risultati della relativa elaborazione numerica del campo di tensione sono riportati in Fig. 2 adimensionalizzati rispetto alla tensione nominale applicata al piatto di spessore maggiore.

Il picco della tensione equivalente non locale risulta massimo in prossimità della radice del cordone di saldatura sul piatto di spessore maggiore esattamente nel punto in cui l'evidenza sperimentale indica il punto di rottura.

\section{Previsione della vita a fatica in giunti complessi tridimensionali}

In generale nei componenti saldati, le cricche nucleano in corrispondenza dei piedi o delle radici dei cordoni cosicché il problema del valore dei carichi da applicare al componente è soprattutto legato all'individuazione del punto di innesco della cricca per fatica. Per mostrare la versatilità del gradiente implicito allo studio della resistenza a fatica di strutture saldate complesse, saranno analizzati quattro differenti tipologie di giunti la cui geometria non può essere ridotta al 2D:

a) giunti con irrigidimento longitudinale (spessore piatto principale 2 o $6 \mathrm{~mm}$ );

b) giunti con irrigidimento circolare (spessore piatto principale $8 \mathrm{~mm}$ );

c) giunti a cordone portante sollecitati a flessione ( spessore piatti $12 \mathrm{~mm}$ );

d) giunti saldati composti da profilati tubolari a sezione rettangolare (spessore profili $7.9 \mathrm{~mm}$ ).

La Fig. 7, a titolo di esempio, mostra il valore della tensione effettiva ottenuta a partire dalla tensione massima principale.

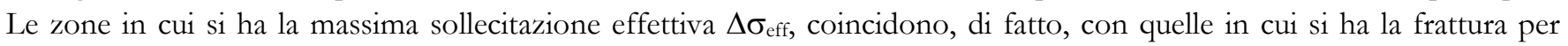
fatica. 
L'accordo fra risultati sperimentali e previsione della vita a fatica è soddisfacente per i giunti delle serie (a) e (b) come evidenziato in Fig. 8. Per le serie (c) e (d) la previsione della vita a fatica appare conservativa (vedere Fig. 9) e ciò potrebbe essere giustificato fondamentalmente per due motivi: 1) nella serie (c) e (d) è presente un carico di torsione nei cordoni di saldatura che comporta la presenza di un modo III singolare assente nelle giunzioni a croce sollecitate a trazione o a flessione; 2) il metodo del gradiente implicito così come è stato proposto in questa sede, è adatto per il calcolo della vita di innesco e di prima propagazione del difetto per fatica. Nel caso di strutture complesse, tale approccio potrebbe risultare in vantaggio di sicurezza nel momento in cui si trascurata completamente la fase di propagazione del difetto. Tuttavia, per quanto riguarda il primo dei due aspetti, gli autori stanno cercando di introdurre nel modello di calcolo del gradiente implicito un approccio di tipo multiassiale alla fatica capace di prendere in considerazione il completo stato di tensione locale.

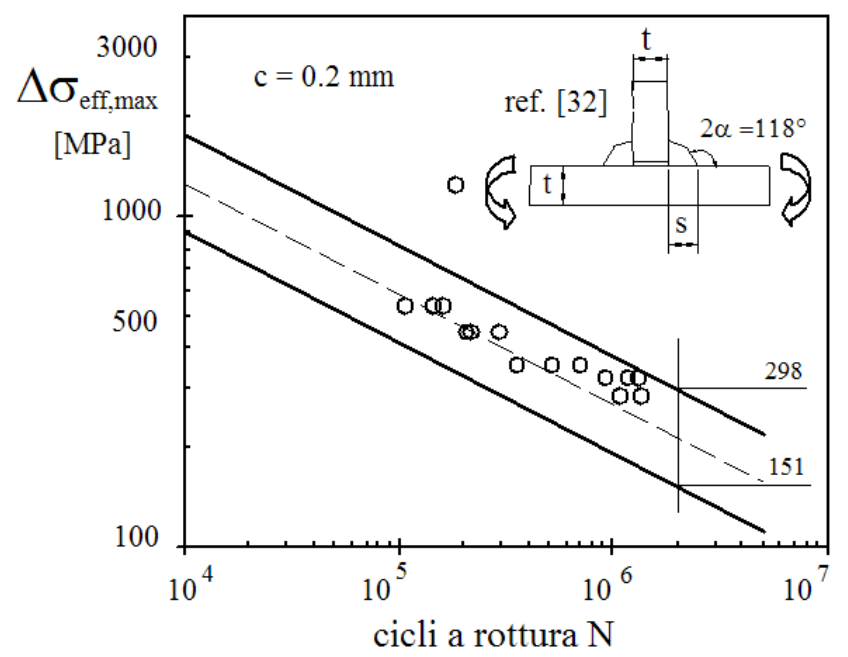

Figura 6: Previsione della resistenza a fatica calcolata per via completamente numerica di una saldatura a $\mathrm{T}$ avente angolo di apertura di $118^{\circ}$ sollecitata a flessione $(\mathrm{t}=10 \mathrm{~mm}, \mathrm{~s}=7.8 \mathrm{~mm}, \mathrm{R}=0.1)$.
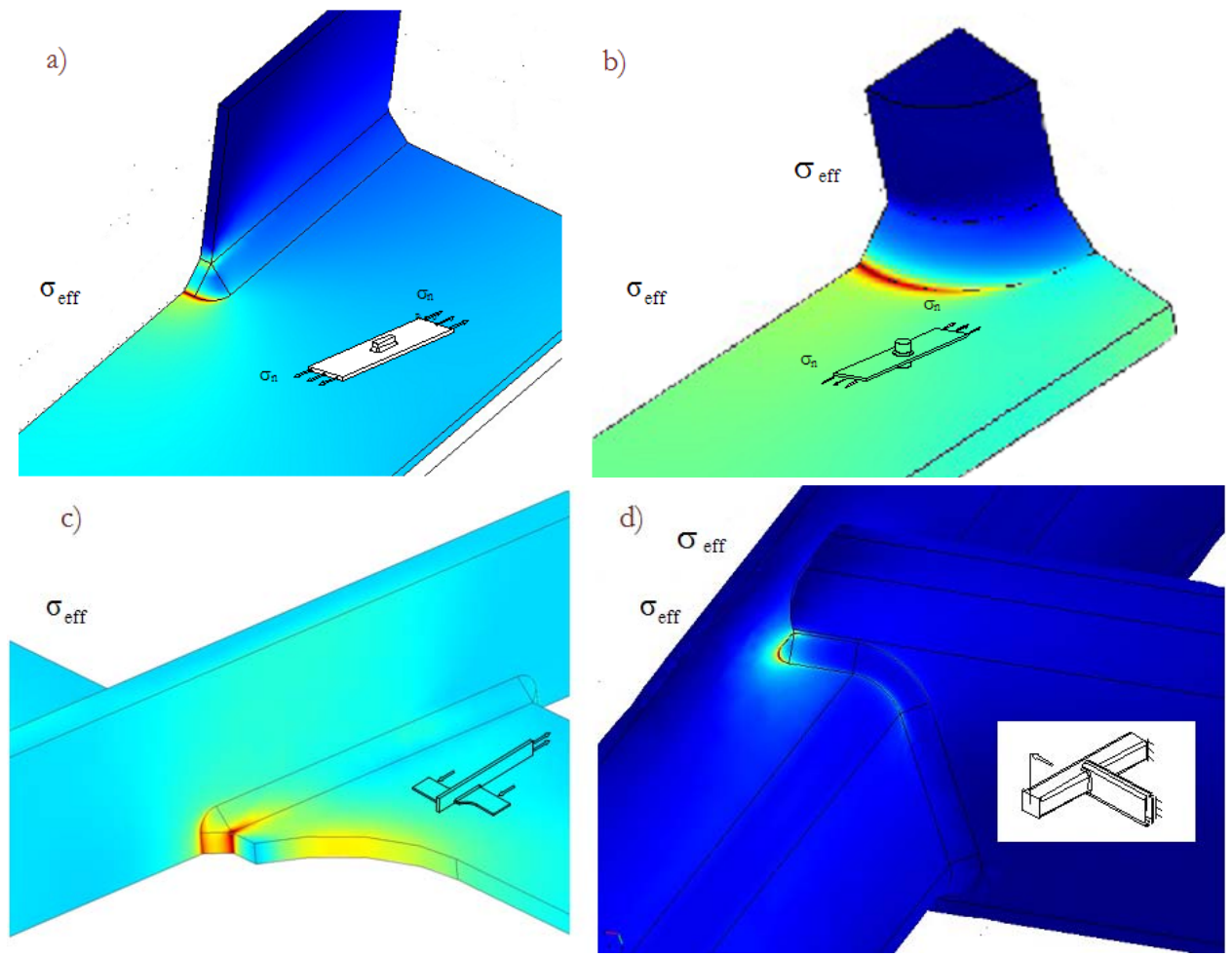

Figure 7: Modelli e risultati ottenuti in termini di tensione effettiva per piastre irrigidite sollecitate a trazione (massima tensione principale come tensione equivalente non locale, $\mathrm{c}=0.2 \mathrm{~mm}$ ). 


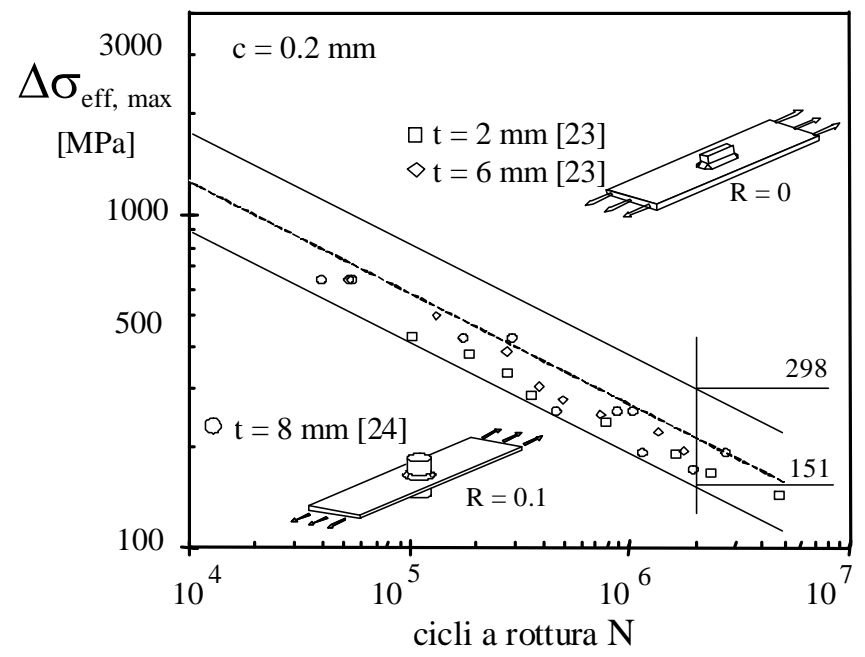

Figura 8: Previsione della vita a fatica di giunti irrigiditi utilizzando il metodo del gradiente implicito (t spessore piatto principale).

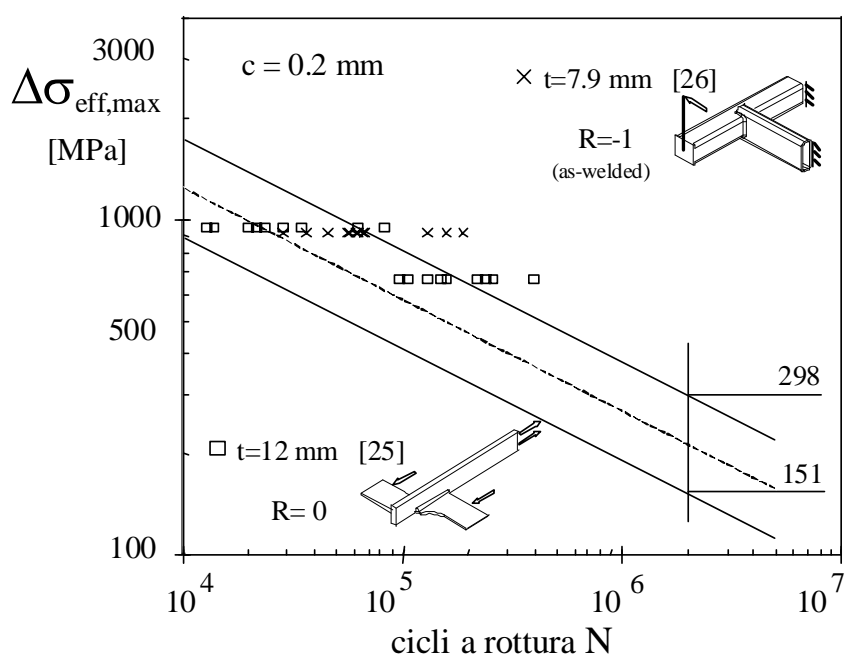

Figura 9: Previsione della vita a fatica di giunti con irrigidimento non simmetrico e di giunti ottenuti con profili tubolari ( $t$ spessore piatto principale).

\section{CONCLUSIONI}

na procedura di verifica a fatica basata sul metodo del gradiente implicito consente di affrontare il problema della singolarità del campo di tensione senza ricorrere a modifiche geometriche del componente in esame o all'impiego di schematizzazioni non lineari del materiale. Disaccoppiando il problema della resistenza dall'effetto gradiente Per durate a fatica superiori a $10^{4}$ cicli, il materiale può essere schematizzato come lineare elastico.

Per giunzioni saldate sollecitate prevalentemente a modo I, il valore del massimo della tensione equivalente non locale, ottenuto dall'analisi numerica, può essere usato come dato di ingresso nella curva di riferimento del comportamento a fatica delle saldature. In questo caso, il metodo del gradiente implicito riporta il calcolo della vita a fatica delle saldature complesse al metodo delle tensioni ammissibili indipendentemente dalla forma del componente in esame.

Per giunzioni sollecitate a modo mosto è necessario l'impiego di una procedura più articolata la quale punto per punto del cordone di saldatura costruisce la curva di Woehler in funzione delle reali condizioni di sollecitazione del corpo.

Il problema delle singolarità tensionali nella progettazione a fatica, in questo articolo, è stato risolto proponendo una filosofia di progettazione basata sui metodi a gradiente implicito capaci di rendere continui i campi di tensione singolari indotti dalla presenza di intagli a spigolo vivo. Tali metodi hanno il vantaggio di poter considerare il materiale come lineare 
elastico trasformando il problema del gradiente tensionale nella risoluzione di una equazione differenziale definita sull'intero corpo in esame. La soluzione trovata, definita come tensione equivalente non locale assume il ruolo di tensione efficace per la valutazione della vita a fatica.

Operando in questo modo è possibile studiare il comportamento a fatica delle giunzioni saldate utilizzando un'unica banda di dispersione, definita in questa sede sulla base di numerose prove sperimentali tratte dalla letteratura.

Il metodo proposto offre il vantaggio di prestarsi per una soluzione completamente numerica del calcolo della vita a fatica delle giunzioni saldate complesse.

\section{BIBLIOGRAFIA}

[1] T. Lassen, Welding Journal 69, Research Supplement, (1990) 75S.

[2] I. Huther, L. Primot, H.P. Lieurade, J.J. Janosch, D. Colchen, S. Debicz, Welding in the World, 35 (2) (1995) 118.

[3] K.A. Macdonald, P.J. Haagensen, Engineering Failure Analysis, 6 (1999) 113.

[4] D. Radaj, C.M. Sonsino, W. Fricke, Fatigue assessment of welded joints by local approaches, Abington Publishing, Abington (2006).

[5] Eurocode 3: Design of steel structures; General rules. 1993-1-1 (1993).

[6] Y. Verreman, B.Nie, Fatigue and Fracture of Engineering Materials and Structures, 19 (1996) 669.

[7] P. Lazzarin, R. Tovo, Fatigue and Fracture of Engineering Materials and Structures, 21 (1998) 1089.

[8] P. Lazzarin, P.Livieri, Int. J. of Fatigue, 23 (2001) 225.

[9] P. Lazzarin, T.Lassen, P. Livieri, Fatigue and Fracture of Engineering Materials and Structures, 26 (2003) 49.

[10] P. Lazzarin, R. Zambardi, Int. J. of Fracture, 112 (2001) 275.

[11] P. Livieri, P. Lazzarin, Int. J. of Fracture, 133 (2005) 247.

[12] R. Tovo, P. Livieri, E. Benvenuti, Int. J. of Fracture, 141 (2006) 497.

[13] R. Tovo, P. Livieri, Engineering Fracture Mechanics, 74 (2007) 515.

[14] R. Tovo, P. Livieri, Engineering Fracture Mechanics Engineering Fracture Mechanics, 75 (7) (2008) 1804.

[15] E. Kroener, Int. J. Sol. Struct., 3 (1967) 731.

[16] C.A. Eringen, D.G.B. Edelen, Int. J. Engng. Science, (1972) 233.

[17] R.H.J. Peerlings, R. de Borst, W.A.M. Brekelmans, J.H.P. de Vree, Int. J. Num. Meth. Engn., 39 (1996) 3391.

[18] M.L. Williams, J.1 of Applied Mechanics, 19 (1952) 526.

[19] P. Lazzarin, R. Tovo, Int. J. of Fracture", 78 (1996) 3.

[20] B. Gross, A. Mendelson, Int. J. of Fracture Mechanics, 8 (1972) 267.

[21] M. H. El Haddad, T.H.Topper, K. N.Smith, ASME, J.of Engineering Material and Technology, 101 (1979) 42.

[22] S. Kainuma, I.T. Kim, Int. J. of Fatigue, 27 (2005) 810.

[23] T.R.Gurney, Fatigue of thin walled joints under complex loading. Abington Publishing, Abington (1997).

[24] L. Susmel, R. Tovo, Int. J. of Fatigue, 28 (2006) 564.

[25] W. Fricke, O. Doerk, Int. J. of Fatigue, 28 (2006) 141.

[26] H Kyuba, P. Dong, Int. J. of Fatigue, 27 (2005) 85. 